\section{TACKLING HEALTH INEQUALITIES: BALANCING UNIVERSAL AND TARGETED APPROACHES}

\section{GUEST EDITORIAL}

\section{Elizabeth Harris}

Centre for Health Equity Training, Research and Evaluation South Western Sydney Area Health Service

\section{Peter Sainsbury}

Division of Population Health

Central Sydney Area Health Service

In 1998-99 expenditure on health services in NSW was estimated at A $\$ 17.6$ billion. This is made up of contributions by the Commonwealth (A $\$ 8.3$ billion), State and Local Governments (A $\$ 4$ billion) and payments through medical benefits funds, personal contributions, and insurance claims, etc. (A $\$ 5.3$ billion). ${ }^{1}$ Many tens of thousands of people work in the health system and in the course of 12 months most people will have had contact with the health system. Health is big business. The challenge we face within the health system when tackling health inequalities is to find ways of harnessing this huge investment of human and economic capital in ways that ensure that our efforts are efficient and effective and not marginalised, with insufficient 'firepower' to make a difference. ${ }^{2}$

One of the most common ways in which this challenge presents itself is in finding the balance between accessible, high quality, universal (whole population) services, and targeted programs that meet the specific needs of vulnerable populations and groups. The development of clinical services for indigenous Australians provides an excellent example. There is growing evidence that community-controlled health services and programs where there is a specific focus on meeting the needs of indigenous communities, contribute significantly to health gain in these communities. ${ }^{3}$ However, it is also true that for most indigenous people, most of the time they will also be using mainstream health services. How should we balance our effort? The evaluation of the first Aboriginal Health Strategic Plan in South Western Sydney Area Health Service found that while all the strategies aimed at building Aboriginal community owned and controlled health services had been either fully ( 54 per cent) or partly ( 46 per cent) achieved, none of the mainstream service strategies had been fully implemented and only 60 per cent had been partly implemented. ${ }^{4}$

continued on page 34

\title{
CONTENTS
}

33 health inequalities: Balancing universal and targeted approaches

35 How can primary care increase equity in health?

38 Can the Families First initiative contribute to reducing health inequalities?

42 The NSW Health Resource Distribution Formula and health inequalities

45 How can evidence-based practice contribute to reducing health inequalities in NSW?

$47 \quad$ Reducing socioeconomic health inequalities: Issues of relevance for policy

50 Building the evidence base to address health inequalities

51 Growing apart: Further analysis of income trends in the 1990s

54 EpiReview:

Cryptosporidiosis in NSW, 1990-2000

58 FactSheet: Pneumococcal disease

59 Communicable Diseases, NSW: March 2002

59 Trends

59 Meningococcal disease among cruise ship passengers

59 Hepatitis A increasing

59 Cryptosporidiosis 
But what is it that we expect of mainstream services and programs? Is there a generic way in which health services can be delivered that will address issues of equity or are there very specific responses that are required for particular groups who have poor health? One way forward is to identify some key service dimensions to be considered in discussing the mix of mainstream and targeted services that we may need. A United Kingdom framework for Primary Health Care research has identified a number of focus areas in the delivery of clinical care systems that may guide this thinking:

- access: attitudes and behaviour of heath professionals and the impact this has on accessibility of services;

- quality of care: timeliness, appropriateness of care, evidence-based practice, comprehensive approaches;

- continuity of care: ongoing management of health problems;

- integration of care: involvement of other service providers in multidisciplinary approaches to care within and across services;

- participatory care: patient-client-carer involvement in decision-making. ${ }^{5}$

The issue is just as real when dealing with population health issues. While it may be possible to ensure that the entire population benefits from some programs such as fluoridation of water, this may not be true for programs aimed at lifestyle change that are dependent on a complex set of behaviours and access to resources. What is the most appropriate mix of universal and targeted health promotion strategies?

Sergerie and Farley have suggested a way through this by developing a set of criteria for designing intervention programs to reduce injury. ${ }^{6}$ Using examples such as bicycle-related head injuries, fire-related injuries, and playground injuries, they make judgements on the relative impact of universal and targeted strategies:

- Is there a difference in the illness rate between socioeconomic groups?

- Are there any differentiating risk factors related to socioeconomic group?

- Are there any effective preventive measures?

- What different interventions exist according to whether a whole population or a selective (high-risk group) approach is used?

- What gains are expected in terms of a reduction of incidence or prevalence according to whether a whole population or a selective approach is used?

- What are the costs and impacts of the measure?

- What ethical principles need to be considered?

The process of asking these questions highlights the difficulties of trying to find relatively simple answers to one aspect of a complex problem. For example, in thinking about reducing fire-related injury the authors identify a lack of smoke detectors, rental accommodation, heating systems, smoking, and alcohol as the main risk factors and recognise that the relative risk of all these factors is higher in low socioeconomic groups. They explore the evidence on whether a population-based (legislative) approach to installing smoke detectors is more effective than the targeted distribution of smoke alarms to high risk communities. However, ultimately it is hard to decide whether either approach to the installation of smoke alarms will by itself be effective while there is a complex web of other risk factors related to the initiation of the fire. In reality a balance between targeted and universal approaches appears most likely to achieve long-term outcomes.

In this third issue of the NSW Public Health Bulletin that focuses on health inequalities, we turn the spotlight towards action to reduce inequalities. The complexity related to balancing on the one hand mainstream and universal interventions and on the other hand targeted interventions is taken up in different ways in the first three papers. Harris and Furler examine ways in which primary care can contribute to reducing health inequalities. They conclude that to be effective specific interventions that provide outreach or targeted preventive services and those that aim to build the capacity of disadvantaged individuals and groups need to be underpinned by a system that ensures good access to high quality health services for everyone.

Alperstein and Nossar examine the ways in which the NSW Families First program can contribute to reducing health inequalities. They provide an overview of the evidence that early intervention and school and community based programs positively impact on child health outcomes. However, they also warn of the limitations of targeting 'reactive services' to high risk families and individuals rather than providing population-based preventive interventions. Many of those who need support may not be identified through tight targeting of services and there is a chance that we may lose sight of the broad global and societal influences that are determining the context in which children are being raised.

Gibbs, Sondalini and Pearce discuss the evolving nature of the NSW Health Resource Distribution Formula (RDF). The tension between providing funds on the basis of health need or health outcomes is discussed and ways in which these considerations can be built into the RDF are outlined. They also discuss the dilemma of providing funds for targeted services within area health services and the difficulties of ensuring that resources are reallocated within area health services to those issues or populations with greatest need.

On a related issue, Leeder provides examples of evidence being used to promote the health of disadvantaged groups 
and argues that currently available information about interventions that actually improve health should be used to help disadvantaged populations now rather than waiting for radical social change. Catchatoor, in an update on the Health Inequalities Research Collaboration, describes how action is being taken at the national level to build the evidence base.

The papers by Turrell and Harding remind us that we need to see any action to tackle health inequalities in the wider social context of income distribution, employment, community infrastructure and global forces. As big as the health system is, it still plays only a small part in the overall solutions. Turrell emphasises the need for action on upstream, midstream and downstream influences on health. Harding, in a sequel to a paper published in the Bulletin last year (Volume 12, Number 5), reminds us that all data sources must be scrutinised carefully.

As our thinking shifts from defining and describing the patterns of health inequality to acting to reduce inequalities, it is important to recognise that doing our 'core business' well and in a way that effectively addresses health inequality is an important contribution to creating a more equal Australian society. The NSW Health and
Equity Statement is a local example of how the priorities of health services are being defined to reduce inequality. ${ }^{7}$

\section{REFERENCES}

1. Australian Institute of Health and Welfare. Health Expenditure No 17. Canberra: AIHW, 2000.

2. King L, Whitecross P. A Health Promotion Perspective: not enough to make you well. Harris E, Sainsbury P, Nutbeam D (editors). Perspectives on Health Inequality. Sydney: Australian Centre for Health Promotion, 2000.

3. Commonwealth Department of Health and Aged Care. Better Health Care-Studies in the successful delivery of health care services for Aboriginal and Torres Strait Islander Australians. Canberra: CDHAC, 2001. Catalogue no. 2942.

4. Carriage C, Harris E, Kristensen E. Evaluation of the first Aboriginal Health Strategic Plan in South Western Sydney. Australian Health Services Review 2000: 23(3): 20-27.

5. National Health Service, Research and Development Strategic Review. Strategic Review Primary Care-Report of the Topic Working Group. London: National Health Service, 1999.

6. Sergerie D, Farley C. The relevance of social inequalities for designing intervention programs. Injury Control and Safety Promotion 2001; 8(3): 155-161.

7. Hyde J. Tackling health inequalities in the NSW Health System: The NSW Heath and Equity Statement. NSW Public Health Bulletin 2001; 12(7): 192-193. 哭

\section{HOW CAN PRIMARY CARE INCREASE EQUITY IN HEALTH?}

\section{Mark Harris}

School of Community Medicine

University of New South Wales

\section{John Furler}

Department of General Practice

University of Melbourne

\section{BACKGROUND}

A number of comparative studies have demonstrated an association between the provision of primary care in developed countries and favourable markers of health status. ${ }^{1,2}$ There is also evidence for an association between health-care systems that are organised around a strong primary-care sector and reduced health inequalities. ${ }^{3}$ Because they reach so much of the population, primary care services such as general practice have an opportunity to address health inequities by improving access to quality care: for example, by providing better anticipatory or preventive care within primary care services themselves, and by outreach into disadvantaged communities. However, to be most effective, these need to be integrated with other multilevel community-based strategies that address the social and economic determinants of health.

\section{ACCESS}

Tudor Hart, working as a general practitioner in Wales, first described the 'inverse care law' in which those with the greatest need access health services the least. ${ }^{4}$ This applies both to access to primary care services and access to those services that occur subsequent to first contact. In Australia, the evidence for disparities in access to primary care is most apparent in relation to primary, secondary, and tertiary preventive care services. People who are socioeconomically disadvantaged are more likely to need, but are less likely to use, preventive health services such as dentists, immunisation, and cancer screening tests. ${ }^{5}$

For example, single parent and migrant families - and families where the parents are unemployed, on low income, or have low levels of education - are at risk of low levels of age-appropriate immunisation..$^{6,7}$ There is evidence to suggest that women of low socioeconomic status are less likely to have attended health services for a Pap smear, although women living in low socioeconomic areas have a higher incidence of cervical cancer. ${ }^{8,9,10}$ This lack of anticipatory care, leading to more crisis management in health, is most evident for indigenous Australians. ${ }^{11,12}$ 